\title{
AMENDMENTS
}

\section{Author Correction: Roles of sumoylation of a reptin chromatin-remodeling complex in cancer metastasis}

Jung Hwa Kim, Hee June Choi, Bogyou Kim, Mi Hyang Kim, Ji Min Lee, Ik Soo Kim, Moon Hee Lee, Soo Joon Choi, Keun II Kim, Su-II Kim, Chin Ha Chung and Sung Hee Baek

Correction to: Nature Cell Biology https://doi.org/10.1038/ncb1415, published online 14 May, 2006.

In the version of this Article originally published, there were several mistakes in Fig $4 \mathrm{~d}$. First, in the 'none' group, as shown by the raw data (UVP000641), lanes 9 and 10 were duplicated and used for both 'anti-Flag' and 'anti-Tip60', and lanes 11 and 12 are the correct data for 'anti-Tip60'. In addition, lanes 2 and 3 were accidently used as 'input', and lanes 7 and 8 are the correct data. Second, in the 'FLAG-SUMO-reptin ${ }^{\mathrm{K} 456 \mathrm{R}}$ ' group, as shown by the raw data (UVP000691), lanes 8 and 9 were mistakenly used for both 'anti-Ac-H3/4' and 'anti-pol II', for which the correct data should be lanes 7-8 and 9-10, respectively. The experiment was repeated three times with similar results. The source data for Fig. $4 \mathrm{~d}$ have been deposited to Figshare (https://figshare.com/s/1d713ae47261fed15c50). The errors have been corrected.
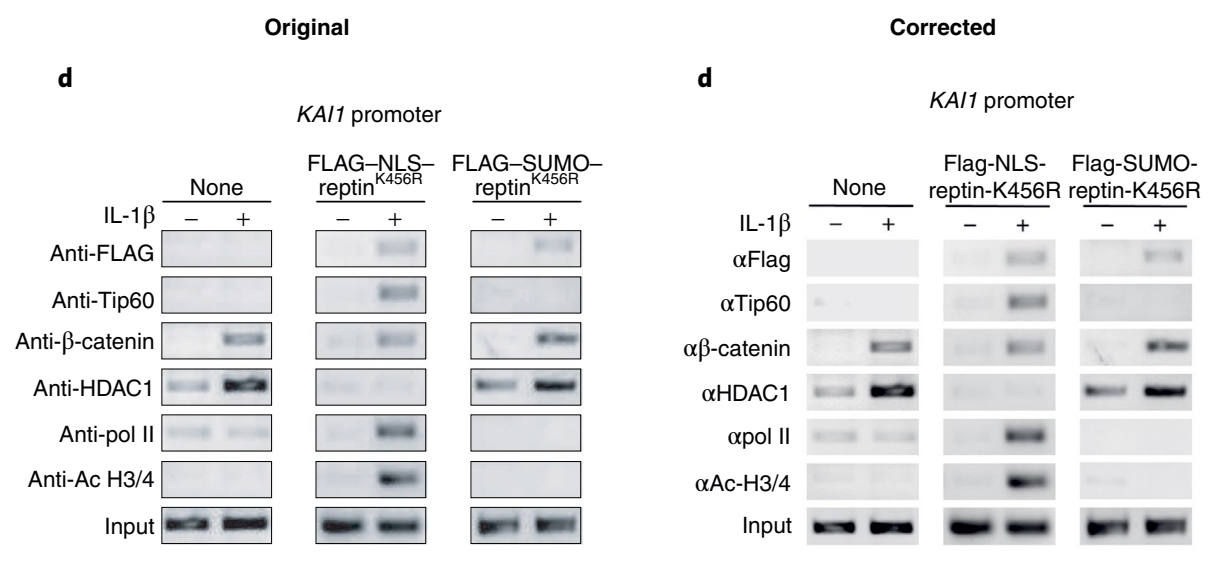

Fig. 1 | Original and corrected.

Published online: 16 June 2021

https://doi.org/10.1038/s41556-021-00715-9

๑) The Author(s), under exclusive licence to Springer Nature Limited 2021 